\title{
Competing responses and the partial reinforcement effect
}

ELVIS C. JONES AND CECIL C. BRIDGES, JR.

TEXAS CHRISTIAN UNIVERSITY

Rats were trained in a runway under consistent and partial reinforcement conditions. The reinforcement groups differed in the number of runway halts and reversals. In most cases the competing behavior conformed to the predictions of competing response theories of the partial reinforcement effect. Spontaneous recovery from competing responses occurred between days.

Competing responses have been invoked by a number of theorists (e.g., Weinstock, 1954; Hulse \& Stanley, 1956; Amsel, 1958) to account for the partial reinforcement effect. Most competing response theorists assume that partially reinforced Ss emit competing responses on unreinforced trials during acquisition. As acquisition continues, however, these responses extinguish or become modified so that they do not readily interfere with the referent response during extinction. Consistently reinforced Ss, however, emit few competing responses during acquisition and the unadapted competing responses are emitted during extinction and interfere with the referent response.

Harris, Smith, \& Weinstock (1962) failed to find significant differences between consistent and partial reinforcement groups' competing responses in a straight runway. However, their response measures were based on lateral deviations en route to the goal box (movements from side to side of the runway). Deviations from an orderly progression down the runway, in the form of halts and reversals, seem more critical in this situation. Also, Harris et al compared performance at the end of acquisition and reacquisition, thus failing to discover trends during the critical, initial trials of acquisition. Further, no data cn extinction were provided. Competing responses produced by partial and consistent reinforcement groups should differ considerably during extinction.

The present experiment dealt with competing responses which directly compete with running, to determine if differences exist between groups which receive consistent and partial reinforcement. Competing response theories have little to say about spontaneous recovery from competing responses, but it might be expected that competing responses become more frequent as a session progresses, and that recovery from these responses occurs between sessions. An attempt was made to determine if such recovery occurs. Method

The Ss were 10 female Sprague-Dawley rats, 100 days old at the start of the experiment. The Ss were assigned to blocks on the basis of weight and then assigned at random to a consistent and partial reinforcement group. The Ss were trained in a Bridges Runway (Bridges, 1966) which was 48 in. long, 4-1/2 in. wide, and 6 in. high, excluding the goal box. The goal box was 10 in. by $4-1 / 2$ in. by 6 in., and contained a copper food cup, 2 in. in diameter and $1 / 2$ in deep. The runway floor was a bar grid.

One set of bars was connected to a common bus-bar and alternate bars were connected between a series of $15,000 \mathrm{ohm}$ resistors. The series of resistors was connected across the output of a $20-v$ regulated power supply. The feet of the Ss provided a path for current to flow from the series of resistors to the bus-bar, and thus the device functioned as a voltage divider, with the S's body acting as the variable contact of a potentiometer. A Sanborn DC amplifier was connected to a Sanborn DC recorder, providing a continuous record of varying voltage levels which were correlated with the Ss' positions in the runway (accurate within $1 \mathrm{in}$.). No noticeable change occurs in the Ss' behavior when the current is turned on or off. Some Ss have been left in the apparatus for several hours with part of the grid disconnected. They spent as much time on the electrified portion as on the nonelectrified portion. Thus, rats probably do not respond to the current.

All Ss were maintained on a $23 \mathrm{hr}$. deprivation schedule in individual home cages. After three days on the schedule, the Ss were given eight acquisition trials per day for three days, followed by three days of extinction. The intertrial interval was $5 \mathrm{~min}$., except that a $20 \mathrm{~min}$. period intervened between the fourth and fifth trial each day. On each trial the $S$ was placed in the start of the runway and the goal box door was closed as soon as the $\mathrm{S}$ entered the goal box. Thirty sec. later, the S was removed to its home cage to await the next trial. There was no food in the home cage until $30 \mathrm{~min}$. following the day's session. Procedures were identical in acquisition and extinction except that no reinforcement was used during extinction. During acquisition, the consistently reinforced (CR) group received five Noyes Precision Food Pellets in the food cup on every trial. The partially reinforced (PR) group, however, found pellets on only $50 \%$ of the acquisition trials (randomly determined).

Tapes from the Sanborn recorder were analyzed to determine the number of halts and reversals in the runway. A halt was defined as the S's staying within a $3 \mathrm{in.} \mathrm{section} \mathrm{of} \mathrm{the} \mathrm{runway} \mathrm{for} 1 \mathrm{sec}$. or more, regardless of the direction of movement prior to the halt. A reversal was defined as a movement of 3 in. or greater away from the goal box when preceded by a movement 


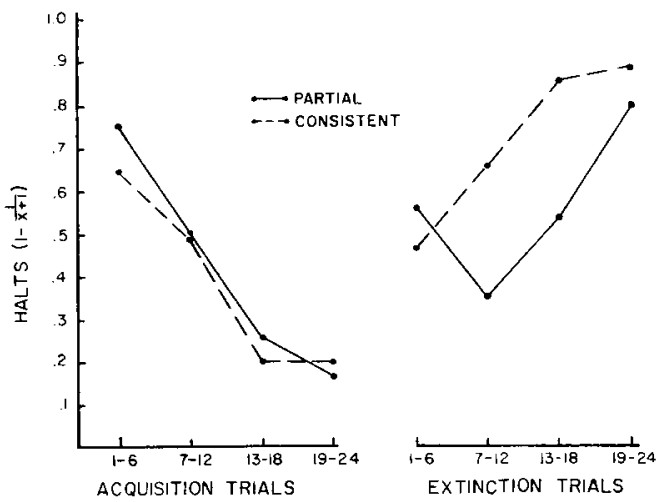

Fig. 1. Runway halts, comparing partial and consistent reinforcement groups.

of $3 \mathrm{in.}$ or more toward the goal box. Movements could be disrupted by halts without affecting the reversal criteria. Scores were converted to $1-\frac{1}{X+1}$ to normalize distributions.

\section{Resulis and Discussion}

As illustrated in Fig. 1, there was little difference in the total number of halts made by the CR and PR groups during acquisition $(p>.10)$. Trend analysis also failed to reveal significant differences between groups (p> .10). During extinction, however, the CR group made more halts than did the PR group $(F=76.10, \mathrm{df}=1 / 7$, $\mathrm{p}<.005)$.

Correlated t-tests were made to determine if spontaneous recovery from halts occurred between days. The difference between the total number of halts on the last trial of each acquisition day and the first trial of the following day (CR and PR groups combined) was significant $(t=2.10, d f=9, p<.05)$. The comparison during extinction, however, was not significant $(p>.05)$. The CR and PR groups did not differ from each other in spontaneous recovery from halts during either acquisition or extinction ( $p>.25$ in both cases).

As can be seen in Fig. 2, reversals by the groups differed during both acquisition and extinction. Despite the small number of Ss in the present study, a definite crossover effect was obtained during acquisition. As would be predicted by most competing response theories, the PR group made more reversals, initially, butby the end of acquisition the CR group made more. Because of the crossover, there was no significant difference in the total number of reversals during acquisition, but trend analysis (over blocks of 6 trials) indicated that the groups had different trends $(F=3.22, \mathrm{df}=3.24, \mathrm{p}<.05)$.

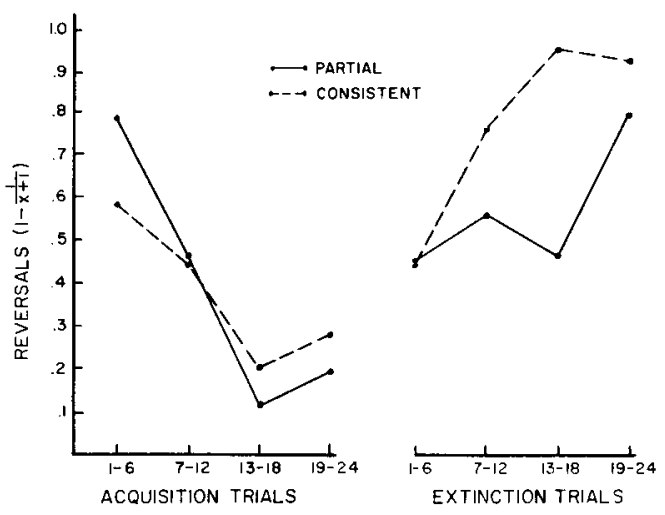

Fig. 2. Reversals of direction in the runway, comparing partial and consistent reinforcement groups.

The difference in the total number of reversals during extinction was significant $(F=33.80, \mathrm{df}=1 / 7, \mathrm{p}<.005)$.

Spontaneous recovery from reversals (CR and PR groups combined) was significant both during acquisition $(t=2.52, d f=9, p<.025)$, and extinction $(t=2.93$, $\mathrm{df}=9, \mathrm{p}<.01$ ). There was no difference between the $\mathrm{CR}$ and $\mathrm{PR}$ groups in spontaneous recovery in either acquisition or extinction ( $p>.10$ in both cases).

Correlations between the combined groups' trial means over the 48 trials of acquisition and extinction were: speed and halts, $r=-.77$, speed and reversals, $r=-.67$, and halts and reversals, $r=.73$ (in each case, $\mathrm{df}=46, \mathrm{p}<.001$ ). Correlations were quite similar in both acquisition and extinction, and did not significantly differ between the groups.

In conclusion, partial reinforcement seems to affect not only running speeds, but also competing responses. The data, with some exceptions, tend to support competing response theories, although it remains to be seen if competing responses play a causal role.

\section{References}

Amsel, A. The role of frustrative nonreward in noncontinuous reward situations. Psychol. Bull., 1958, 55, 102-119.

Bridges, C. C. All-electric recording in a straight alley. Amer. J. Psychol., 1966, 79, 131-133.

Harris, S. J., Smith, M. G., \& Weinstock, S. Effects of nonreinforcement on subsequent reinforced running behavior. J.exp. Psychol., $1962,64,188-198$.

Hulse, S. H., Jr., \& Stanley, W. C. Extinction by omission of food as related to partial and secondary reinforcement. J. exp. Psychol., 1956, 52, 221-227.

Weinstock, $\mathbf{S}$. Resistance to extinction of a running response following partial reinforcement under widely spaced trials. $J$. comp. physiol. Psychol., 1954, 47, 318-322. 